\title{
Performance of the Barrett Toric Calculator with and without measurements of posterior corneal curvature
}

\author{
Janusz Skrzypecki ${ }^{1,2} \cdot$ Menka Sanghvi Patel ${ }^{3} \cdot$ Leejee H. Suh ${ }^{3}$
}

Received: 30 January 2019 / Revised: 15 April 2019 / Accepted: 24 April 2019 / Published online: 12 June 2019

(c) The Author(s), under exclusive licence to The Royal College of Ophthalmologists 2019

\begin{abstract}
Background Toric intraocular lens power calculators, e.g., the Barrett Toric Calculator, based on predicted, rather than on measured posterior corneal curvature have yielded the best results so far. However, recent update of the Barrett Toric Calculator aims to fine tune its refractive predictions with the input of measured posterior corneal curvature. Here, we wanted to compare refractive predictions of the Barrett Toric Calculator, based on IOL Master 700 biometry, with and without measurements of posterior corneal curvature.

Methods In total 30 eyes were included in the study. One-month postoperative manifest refraction and predicted residual refractive error of both formulas were utilized to calculate mean absolute error and centroid error in predicted residual astigmatism. The Pentacam was used to measure posterior corneal curvature.

Results We did not find any statistically significant difference in mean absolute error and centroid error in predicted residual astigmatism between the Barrett Toric Calculator with and without measurement of posterior corneal curvature. Post-hoc analysis of with-the-rule and against-the-rule astigmatic eyes did not reveal any significant differences as well.

Conclusions Astigmatism prediction errors, based on IOL Master 700 biometry, with and without measured posterior corneal curvature, were similar. To the best of our knowledge, the updated Barrett Toric Calculator is the first formula to provide non-inferior and reliable predictions based on measurement of posterior corneal curvature.
\end{abstract}

\section{Introduction}

Cataract surgery is the most performed surgery in the United States. With the aging population, cataract surgical numbers are steadily increasing. Calculation of the intraocular lens (IOL) power remains one of the most important steps in the preoperative evaluation for cataract surgery [1]. Many formulas were developed for precise spherical power calculations, including the Barrett Universal II formula, the Hill-RBF formula or the Olsen formula [2-4].

$\triangle$ Leejee H. Suh

lhs2118@cumc.columbia.edu

1 Department of Ophthalmology, Medical University of Warsaw, Warsaw, Poland

2 Department of Experimental Physiology and Pathophysiology, Laboratory of Centre for Preclinical Research, Medical University of Warsaw, Warsaw, Poland

3 Department of Ophthalmology, Columbia University College of Physicians and Surgeons, New York, NY, USA
Nevertheless, a significant number of patients with corneal astigmatism do not achieve satisfactory uncorrected distance visual acuity (UDVA) with standard monofocal IOLs [5]. Monofocal toric IOLs, which were developed to fill this gap, require a slightly different approach to IOL power calculations [6]. Estimation of the cylindrical power of the toric IOL is based on the total corneal astigmatism (TCA), a vectorial sum of anterior corneal astigmatism (ACA) and posterior corneal astigmatism (PCA) [6]. Currently, no device measures posterior corneal curvature with complete precision; therefore, some theoretical assumptions are undertaken by these calculators [7]. It is believed that the measurement of ACA, regardless of its axis, could be approximated to the TCA by a fudge factor, i.e., the refractive index of 1.3375. However, it was soon recognized that PCA has a significant axis-dependent effect on the TCA [8]. Therefore, this approach could lead to an overestimation of the with-the-rule (WTR) astigmatism and an underestimation of the against-the-rule (ATR) astigmatism [8]. The Baylor nomogram and ray tracing addressed this issue with mixed results $[9,10]$. Some formulas have incorporated measurements of the posterior corneal 
curvature to fine tune their predictions [11]. Despite the numerous methods available, recent studies show that the Barrett Toric formula outperforms other calculators [12]. The standard version of the Barrett Toric Calculator includes an unpublished algorithm to predict effective lens position (ELP) and PCA, both essential for precise toric IOL calculations. Recently authors of the formula released an enhanced version of the calculator by the adding the capability to input measured PCA, which in theory, should further improve refractive outcomes [13]. Additionally, previous studies have shown that the type of biometry or keratometry might have an influence on performance of the Barrett Toric Calculator. The PCA measurements obtained by several devices are compatible with the enhanced calculator, including IOL Master 700 (Zeiss, Germany), Pentacam (Oculus, Germany) or Gallilei (Ziemer, Germany) [13]. We used IOL Master 700 measurements, a novel swept-source OCT (optical coherence tomography) based biometer. We wanted to compare refractive predictions of the Barrett Toric Calculator with and without utilization of PCA measurements.

\section{Materials and methods}

In total 30 eyes of 24 patients who underwent uneventful cataract extraction and toric intraocular lens implantation were included in this retrospective study from January 2016 to April 2018 at the Columbia University Medical Center (CUMC, New York, USA). The study was approved by the Institutional Review Board of Columbia University.

Patients with age-related cataracts and preoperative astigmatism of at least 1.0 D for against-the-rule (ATR) and 1.2 D for with-the-rule (WTR) astigmatism by topographic Scheimpflug analysis (Oculus Pentacam, Germany) were included in the study. Patients with ocular or systemic diseases, which could affect best-corrected visual acuity (BCVA) or ELP, e.g. pseudoexfoliation syndrome, glaucoma, macular degeneration and diabetes, were excluded. Furthermore, any surgical complication or lens misalignment would exclude patient from the study.

Preoperative best-corrected visual acuity (BCVA) was assessed with Snellen charts. Routine anterior and posterior segment examination was performed prior to surgery. Topographic Scheimpflug corneal topography (Oculus Pentacam, Germany) was utilized to screen for irregular astigmatism (exclusion criterion) and to measure PCA. Finally, all patients underwent optical biometry with IOL Master 700 (Zeiss, Germany) and toric IOL (AcrySof Toric) power were selected based on the standard Barrett Toric Calculator with predicted PCA [13].

Patients had the implantation axis marked in the preoperative suite with RoboMarker (Surgilum, USA). To avoid bias of surgically induced astigmatism (SIA), only surgeries performed by one experienced cataract and corneal surgeon (L.S.) were included. A clear corneal incision was made temporally with an estimated surgeon specific induced astigmatism (SIA) of 0.2 was created with a $2.2 \mathrm{~mm}$ keratome on all patients. Following routine phacoemulsification, a monofocal toric IOL (AcrySof Toric) was implanted and aligned with the pre-marked axis. All patients were examined postoperatively day one, week one and month one. The BCVA, lens position, including axis and tilt, were assessed at the slit lamp following mydriasis. Axis of the IOL was assessed with toriCAM (Graham Barrett, AppStore, USA).

For the purpose of the study, one-month manifest refraction at the spectacle plane was considered a final refractive outcome of the toric IOL implantation [14, 15]. The difference between the one month manifest refraction and predicted residual refractive error according to the Barrett Toric formula with predicted PCA was calculated. Subsequently, we calculated the difference between manifest refraction and predicted residual refractive error with the enhanced Barrett Toric formula, which included the input of measured PCA. The PCA measured by topographic Scheimpflug (Oculus Pentacam, Germany) was utilized in this study. Finally, these values were evaluated to ascertain whether the measurement of PCA improves the Barrett Toric Calculator refractive predictions. Mean absolute error (MAE) and centroid error in predicted residual astigmatism were used to compare formulas. Centroid error, which assesses both vectors of astigmatism, was calculated according to Holladay [16]. Vector analysis was utilized when necessary. Additionally, a separate, post-hoc analysis for WTR and ATR astigmatism was performed.

The power of the study was set at $80 \%$ with a significance level at $5 \%$ were set to detect a difference in predicted residual astigmatism at the level of $0.25 \pm 0.5 \mathrm{D}$, an amount we considered to be clinically significant. Shapiro-Wilk test was used to screen for normal distribution of data. Parametric comparisons were performed with the paired Student's $t$-test, whereas non-parametric calculations were made with the Wilcoxon rank-sum test. The Bonferrroni correction was applied for multiple comparisons. All calculations were performed with Excel (Microsoft, USA) or Graphpad Prism 7 (Graphpad Software, USA). Mean absolute error and centroid error were evaluated using the Barrett Toric Calculator comparing predicted versus measured posterior corneal curvature, values that were obtained from topographic Scheimpflug analysis (Oculus Pentacam, Germany). One month postoperative manifest refraction was compared to predicted residual refractive error obtained from the Barrett Toric Calculator using predicted versus measured posterior corneal curvature measurements. 


\section{Results}

In total 30 eyes of 24 patients were included in our study. Table 1 presents baseline characteristics of the group. There was no statistically significant difference in MAEs in predicted astigmatism obtained with both versions of the Barrett Toric calculator, with predicted and measured PCA (Table 2). Furthermore, vector analysis did not reveal any significant differences in centroid errors in predicted residual astigmatism between the two calculators [the Barrett Toric calculator with predicted PCA: $x=0.12 \pm 0.4, y=$ $-0.08 \pm 0.31$; the Barrett Toric calculator with measured PCA: $0.12 \pm 0.33, y=-0.07 \pm 0.35, p>0.05]$. Figure 1

Post-hoc analysis did not reveal any statistically significant differences in MAE or centroid error in eyes with WTR astigmatism (the Barrett Toric calculator with predicted PCA: $x=0.044 \pm 0.26, y=-0.14 \pm 0.32$; the Barrett Toric calculator with measured PCA: $0.09 \pm 0.35, y=$ $-0.15 \pm 0.4 ; p>0.05$ ) and ATR astigmatism (the Barrett

Table 1 Baseline characteristics of the studied group

\begin{tabular}{ll}
\hline Parameter & No./Value \pm SD (range) \\
\hline No. of patients & 24 \\
No. of eyes & 30 \\
No. of eyes with WTR astigmatism & 13 \\
No. of eyes with ATR astigmatism & 12 \\
No. of eyes with oblique astigmatism & 5 \\
Flat meridian (IOL Master K) & $43.51 \pm 2.26 \mathrm{D}$ \\
& $(41.58,49.08)$ \\
Steep meridian (IOL master K) & $45.55 \pm 2.21 \mathrm{D}(41.9,50)$ \\
Axial length & $24.93 \pm 1.99 \mathrm{~mm}$ \\
& $(21.97,30.83)$ \\
Anterior chamber depth & $3.3 \pm 0.43 \mathrm{~mm}(2.42,3.86)$ \\
Lens thickness & $4.57 \pm 0.38 \mathrm{~mm}(3.9,5.4)$ \\
White-to-white & $12.11 \pm 0.39(11.3,12.9)$ \\
Flat meridian, posterior corneal & $6.61 \pm 0.50 \mathrm{~mm}(5.84,8.83)$ \\
curvature (Pentacam) & \\
Steep meridian, posterior corneal & $6.12 \pm 0.32 \mathrm{~mm}(5.54,6.86)$ \\
curvature (Pentacam) &
\end{tabular}

All results are expressed as a number or mean absolute average \pm standard deviation (SD). Range is given in brackets

Table 2 Mean absolute error (MAE) and centroid error in predicted residual astigmatism for the Barrett Toric calculator with predicted posterior corneal astigmatism (PCA) and the Barrett Toric calculator with measured PCA

\begin{tabular}{lll}
\hline & $\begin{array}{l}\text { Barrett Toric, } \\
\text { predicted PCA }\end{array}$ & $\begin{array}{l}\text { Barrett Toric, } \\
\text { measured PCA }\end{array}$ \\
\hline MAE & $0.33 \pm 0.15 \mathrm{D}$ & $0.31 \pm 0.23 \mathrm{D}$ \\
centroid error & $0.14 \pm 0.35 \mathrm{D} 164^{\circ}$ & $0.13 \pm 0.35 \mathrm{D} 163^{\circ}$
\end{tabular}

Results are expressed as mean \pm standard deviation
Toric calculator with predicted PCA: $x=0.14 \pm 0.34, y=$ $-0.06 \pm 0.15$; the Barrett Toric calculator with measured PCA: $0.23 \pm 0.28, y=-0.07 \pm 0.14 ; p>0.05)$. Table 3 , Figs. 2 and 3

\section{Discussion}

We found that the Barrett Toric Calculator based on predicted PCA precisely calculates toric IOL power. Utilization of PCA measurements, which was facilitated by the latest update of the calculator, produced similar residual astigmatism predictions in comparison to the classic version of the Barrett Toric Calculator, which uses a predicted PCA. There was no statistically significant difference in MAEs in predicted astigmatism obtained with both versions of the Barrett Toric calculator i.e. with predicted and measured PCA. In our opinion, this is the first attempt to include measurements of PCA in toric IOL calculations, which demonstrated non-inferior results when compared to using the classic Barrett Toric Calculator with the predicted PCA.

The goal of having only one calculator to provide the ideal refractive outcome has always been a quest in modern cataract surgery $[1,17]$. Approximately $20-30 \%$ of patients who undergo cataract surgery have a preoperative corneal astigmatism of at least 1.0-1.5D and would benefit from the implantation of a toric IOL [18]. Shimizu et al. were the first to report application of the non-foldable toric IOL. Since then, numerous approaches have been developed to calculate cylindrical power of the IOL [18]. However, before recognition of the importance of the variability of PCA, refractive surprises following toric IOL implantation were relatively frequent $[18,19]$.

The first version of the Alcon Toric calculator, as well as the Holladay Toric calculator used a fixed ratio of anterior and posterior curvature to obtain required toric correction at the corneal plane [20]. Due to their inaccuracy in predicting TCA, these methods yielded high MAE and centroid error in predicted residual astigmatism, up to $0.8 \mathrm{D} \pm 0.4$ and $0.56 \mathrm{D} @ 180$ respectively [20]. As soon as Koch et al. underscored that PCA has different effect on WTR and ATR keratometric astigmatism [8], the Alcon Toric calculator was updated with the Baylor nomogram [21]. The MAE and centroid error in residual astigmatism decreased slightly; however, some studies did not show statistically significant differences [21]. Subsequently, other nomograms e.g. the Abulafia-Koch formula, were developed to improve refractive outcomes following toric IOL implantation [14]. These formulas were evaluated by various authors and some reported MAEs and centroid errors close to zero $[14,22]$. However, only the Barrett Toric Calculator has consistently shown to outperform the other formulas and produce low errors in predicted residual astigmatism 
Fig. 1 Double-angle diagrams illustrating centroid errors in predicted residual astigmatism for the Barrett Toric calculator with a predicted posterior corneal astigmatism and b measured posterior corneal astigmatism (PCA)

\section{A. Barrett Toric calculator with predicted PCA centroid error: $0.14 \pm 0.35 \mathrm{D} 164^{\circ}$ $\mathrm{x}=0.12 \pm 0.40 \mathrm{y}=-0.08 \pm 0.31$}

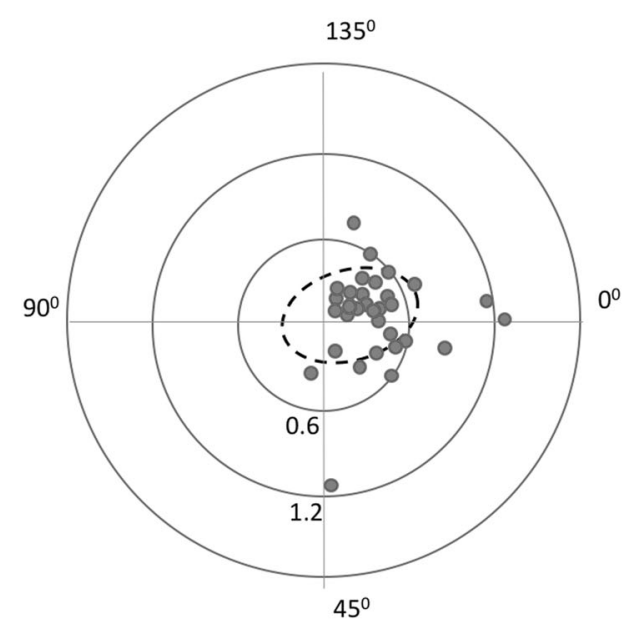

(-) - standard deviation (SD)
B. Barrett Toric calculator with measured PCA centroid error: $0.13 \pm 0.35 \mathrm{D} 163^{\circ}$ $0,12 \pm 0.30, y=-0,07 \pm 0.35$

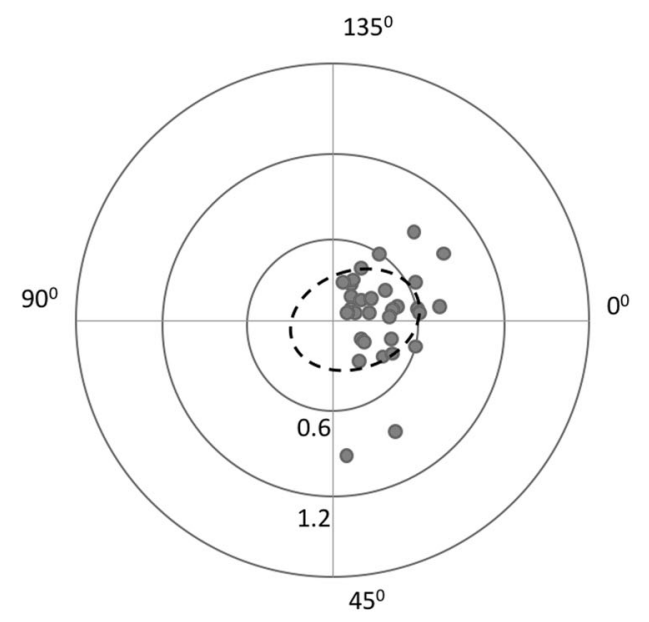

Table 3 Post-hoc analysis of eyes with keratometric against-the-rule (ATR) and with-the-rule (WTR) astigmatism

\begin{tabular}{lll}
\hline & $\begin{array}{l}\text { Barrett Toric, } \\
\text { predicted PCA }\end{array}$ & $\begin{array}{l}\text { Barrett Toric, } \\
\text { measured PCA }\end{array}$ \\
\hline $\begin{array}{l}\text { WTR astigmatism } \\
\text { MAE }\end{array}$ & $0.29 \pm 0.2$ & $0.27 \pm 0.2$ \\
$\begin{array}{l}\text { centroid error } \\
\text { ATR astigmatism }\end{array}$ & $0.14 \pm 0.29144^{\circ}$ & $0.18 \pm 0.37151^{\circ}$ \\
MAE & $0.28 \pm 0.12 \mathrm{D}$ & $0.29 \pm 0.24 \mathrm{D}$ \\
centroid error & $0.14 \pm 0.23 \mathrm{D} \mathrm{178^{ \circ }}$ & $0.24 \pm 0.21 \mathrm{D} \mathrm{168^{ \circ }}$ \\
\hline
\end{tabular}

Mean absolute error (MAE) and centroid error in predicted residual astigmatism for the Barrett Toric calculator with predicted posterior corneal astigmatism (PCA) and the Barrett Toric calculator with measured PCA

[20-22]. Similarly, previous studies of the Barrett Toric calculator did not show any significant difference in prediction errors of WTR and ATR astigmatism [20]. The results of our study are in line with these reports.

Some studies have highlighted that centroid error predicted by the Barrett Toric Calculator might differ relative to the type of utilized keratometry [12]. A 32-point Lenstar keratometry was shown to be slightly more accurate than the 6-point IOL Master 500 keratometry [12]. However, this discrepancy does not appear to be clinically significant as MAE in predicted residual astigmatism is similar between the two devices [12]. The K-values utilized in our study were obtained with 28-point keratometry, IOL Master 700 biometry (Zeiss, Germany). Although, we are unable to conduct precise statistical analysis, it seems that results produced with the IOL Master 700 overlap with previous reports.
Numerous attempts have been made to incorporate measurement of posterior corneal curvature into calculation of the toric IOL power [12], including studies that involved adjusting keratometry by topographic Scheimpflug analysis (Oculus Pentacam, Germany) True K values. However, as IOL power formulas have some built-in corrections to approximate keratometry readings to real corneal power, any additional manual fine-tuning leads to skewed results [20].

Our results have shown that inclusion of measured PCA in the Barrett Toric Calculator produces non-inferior results to the classic formula with predicted PCA. Furthermore, we hypothesize that this upgrade might lead to improved refractive predictions in the setting when posterior curvature cannot be directly predicted from the anterior curvature, e.g. keratoconus [23].

Results of our study are limited by a small group of patients. An increased number of participants would improve the power of the study. Furthermore, the Barrett Toric Calculator allows an input of posterior corneal curvature measurements obtained from various devices [13]. Given that previous studies have reported significant discrepancies between measurement modalities of posterior cornea curvature, analysis of astigmatism prediction based on optical coherence tomography or ray-tracing might be of additional value [7].

It should be also discussed that absolute value of centroid error is affected by SIA. Here, we utilized SIA of 0.2, which is higher than the most often reported amount of astigmatism induced by $2.2 \mathrm{~mm}$ temporal incision, i.e. 0.1 [24]. However, considering that the same value of SIA was input into both versions of the calculator, the amount of SIA 
Fig. 2 Double-angle diagrams illustrating centroid errors in predicted residual astigmatism in eyes with against-the-rule (ATR) astigmatism for the Barrett Toric calculator with a predicted posterior corneal astigmatism and $\mathbf{b}$ measured posterior corneal astigmatism (PCA)
Fig. 3 Double-angle diagrams illustrating centroid errors in predicted residual astigmatism in eyes with with-the-rule (WTR) astigmatism for the Barrett Toric calculator with a predicted posterior corneal astigmatism and $\mathbf{b}$ measured posterior corneal astigmatism (PCA)

\section{ATR astigmatism}

A. Barrett Toric calculator with predicted PCA centroid error: $0.14 \pm 0.23 \mathrm{D} 178^{\circ}$ $x=0.14 \pm 0.34 y=-0.06 \pm 0.15$

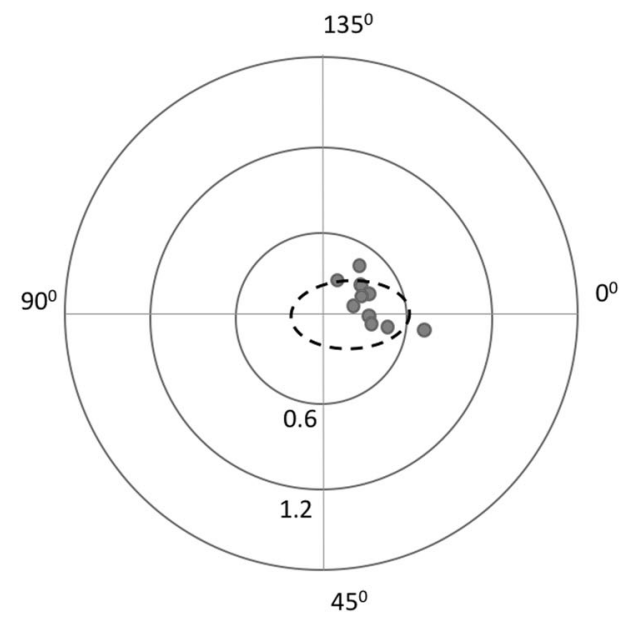

$45^{0}$
B. Barrett Toric calculator with measured PCA centroid error: $0.25 \pm 0.22 \mathrm{D} 168^{\circ}$ $X=0.23 \pm 0.28 y=-0.07 \pm 0.14$

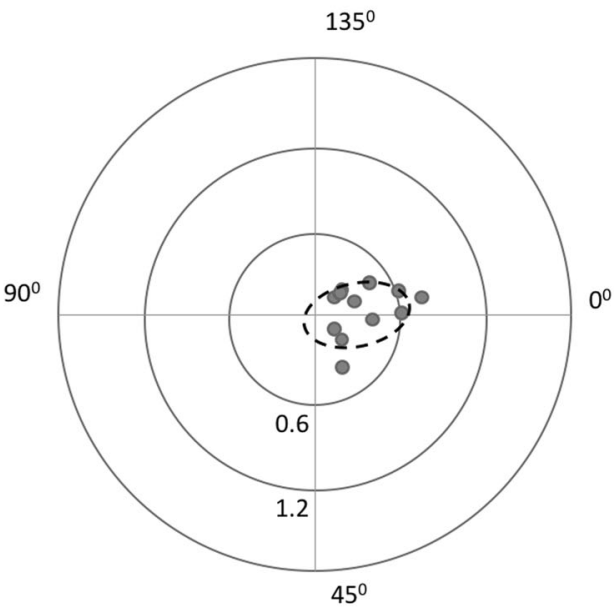

- - standard deviation (SD)

\section{WTR astigmatism}

A. Barrett Toric calculator with predicted PCA centroid error: $0.14 \pm 0.29144^{\circ}$ $x=0.04 \pm 0.26 y=-0,14 \pm 0.32$

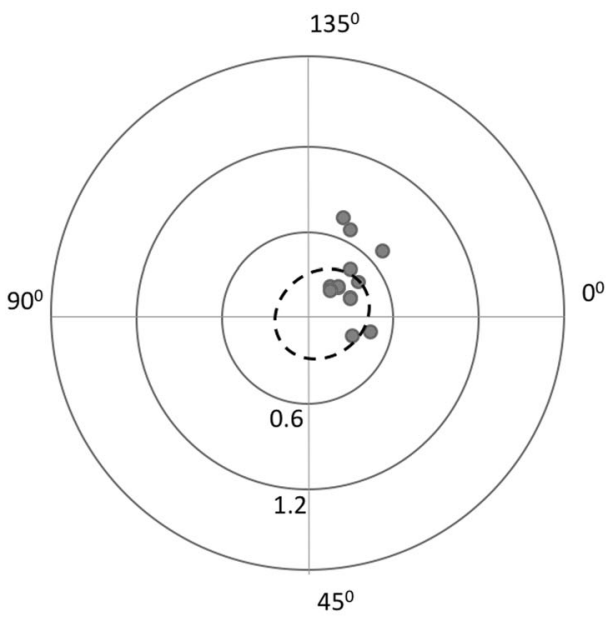

- - standard deviation (SD)
B. Barrett Toric calculator with measured PCA centroid error: $0.18 \pm 0.37151^{0}$ $\mathrm{X}=0.09 \pm 0.35 \mathrm{y}=-0.15 \pm 0.4$

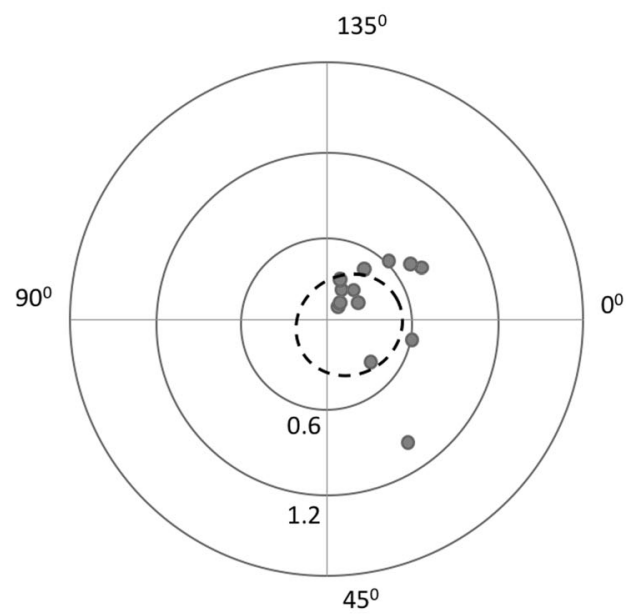

should have no significant influence on the comparison between two formulas.

In conclusion, the Barrett Toric Calculator, based on an IOL Master 700 biometry, produces low prediction errors. Furthermore, calculations with and without PCA measurements (Oculus Pentacam, Germany) produce similar refractive predictions. More studies are needed to show potential superiority of the updated Barrett Toric calculator in the setting of unusual ratio of anterior and posterior corneal curvature.

\section{Summary}

\section{What was known before}

- The Barrett Toric Calculator, based on predicted rather than on measured posterior corneal curvature, is one of more precise calculators for toric intraocular lens power calculation. Performance of the recent update of the Barrett Toric Calculator, which allows for input of 
measured posterior corneal curvature, has not been compared with its predecessor so far.

\section{What this study adds}

- The update of the Barrett Toric Calculator produces non-inferior predictions in normal eyes in comparison to its previous version. The updated Barrett Toric Calculator might be a robust tool in eyes with skewed anterior to posterior corneal curvature ratio.

\section{Compliance with ethical standards}

Conflict of interest The authors declare that they have no conflict of interest.

Publisher's note: Springer Nature remains neutral with regard to jurisdictional claims in published maps and institutional affiliations.

\section{References}

1. Olsen T. Calculation of intraocular lens power: a review. Acta Ophthalmol Scand. 2007;85:472-85.

2. Cooke DL, Cooke TL. Comparison of 9 intraocular lens power calculation formulas. J Cataract Refract Surg. 2016;42:1157-64.

3. Roberts TV, Hodge C, Sutton G, Lawless M, contributors to the Vision Eye Institute IOLor. Comparison of Hill-radial basis function, Barrett Universal and current third generation formulas for the calculation of intraocular lens power during cataract surgery. Clin Exp Ophthalmol. 2018;46:240-6.

4. Koch DD, Hill W, Abulafia A, Wang L. Pursuing perfection in intraocular lens calculations: I. Logical approach for classifying IOL calculation formulas. J Cataract Refract Surg. 2017;43:717-8.

5. Visser N, Beckers HJ, Bauer NJ, Gast ST, Zijlmans BL, Berenschot TT, et al. Toric vs aspherical control intraocular lenses in patients with cataract and corneal astigmatism: a randomized clinical trial. JAMA Ophthalmol. 2014;132:1462-8.

6. Reitblat O, Levy A, Kleinmann G, Abulafia A, Assia EI. Effect of posterior corneal astigmatism on power calculation and alignment of toric intraocular lenses: comparison of methodologies. J Cataract Refract Surg. 2016;42:217-25.

7. Koch DD. The posterior cornea: hiding in plain sight. Ophthalmology. 2015;122:1070-1.
8. Koch DD, Ali SF, Weikert MP, Shirayama M, Jenkins R, Wang L. Contribution of posterior corneal astigmatism to total corneal astigmatism. J Cataract Refract Surg. 2012;38:2080-7.

9. Koch DD, Jenkins RB, Weikert MP, Yeu E, Wang L. Correcting astigmatism with toric intraocular lenses: effect of posterior corneal astigmatism. J Cataract Refract Surg. 2013;39:1803-9.

10. Hoffmann PC, Wahl J, Hutz WW, Preussner PR. A ray tracing approach to calculate toric intraocular lenses. J Refract Surg. 2013;29:402-8.

11. Preussner PR, Hoffmann P, Wahl J. Impact of posterior corneal surface on toric intraocular lens (IOL) calculation. Curr Eye Res. 2015;40:809-14.

12. Abulafia A, Barrett GD, Kleinmann G, Ofir S, Levy A, Marcovich $\mathrm{AL}$, et al. Prediction of refractive outcomes with toric intraocular lens implantation. J Cataract Refract Surg. 2015;41:936-44.

13. http://www.ascrs.org/barrett-toric-calculator. Accessed 15.01.2018.

14. Abulafia A, Koch DD, Wang L, Hill WE, Assia EI, Franchina M, et al. New regression formula for toric intraocular lens calculations. J Cataract Refract Surg. 2016;42:663-71.

15. Hoffmann PC, Abraham M, Hirnschall N, Findl O. Prediction of residual astigmatism after cataract surgery using swept source fourier domain optical coherence tomography. Curr Eye Res. 2014;39:1178-86.

16. Holladay JT, Moran JR, Kezirian GM. Analysis of aggregate surgically induced refractive change, prediction error, and intraocular astigmatism. J Cataract Refract Surg. 2001;27:61-79.

17. Dang MS, Raj PP. SRK II formula in the calculation of intraocular lens power. Br J Ophthalmol. 1989;73:823-6.

18. Visser N, Bauer NJ, Nuijts RM. Toric intraocular lenses: historical overview, patient selection, IOL calculation, surgical techniques, clinical outcomes, and complications. J Cataract Refract Surg. 2013;39:624-37.

19. Sun XY, Vicary D, Montgomery P, Griffiths M. Toric intraocular lenses for correcting astigmatism in 130 eyes. Ophthalmology. 2000;107:1776-81. discussion 1781-1772.

20. Abulafia A, Hill WE, Franchina M, Barrett GD. Comparison of methods to predict residual astigmatism after intraocular lens implantation. J Refract Surg. 2015;31:699-707.

21. Ferreira TB, Ribeiro P, Ribeiro FJ, O'Neill JG. Comparison of astigmatic prediction errors associated with new calculation methods for toric intraocular lenses. J Cataract Refract Surg. 2017;43:340-7.

22. Ferreira TB, Ribeiro P, Ribeiro FJ, O'Neill JG. Comparison of methodologies using estimated or measured values of total corneal astigmatism for toric intraocular lens power calculation. J Refract Surg. 2017;33:794-800.

23. Thebpatiphat N, Hammersmith KM, Rapuano CJ, Ayres BD, Cohen EJ. Cataract surgery in keratoconus. Eye Contact Lens. 2007;33:244-6.

24. Masket S, Wang L, Belani S. Induced astigmatism with 2.2- and 3.0-mm coaxial phacoemulsification incisions. J Refract Surg. 2009;25:21-24. 